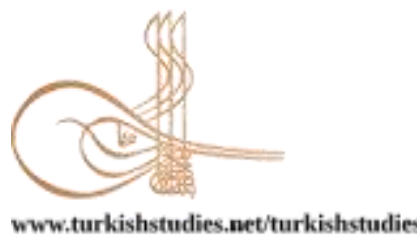

Turkish Studies

www.turkishstudies.netturkishstudies

eISSN: $1308-2140$

\title{
Zwischen Virtueller Realität und Alternativen Fakten Fake News und Verschwörungstheorien in den Sozialen Medien
}

Between Virtual Reality and Alternative Facts- Fakenews and Conspiracy Theories in the Social Media

\author{
Zehra Özkeçeci*
}

\begin{abstract}
This article deals with the spread of fake news and conspiracy theories in the digital age. The social media make it easier to publish every realistic and absurd theory worldwide without great financial effort and academic prerequisites. Although there have always been successful attempts in history to spread false reports and rumours, today's technological advances make it possible to reach a much wider audience in real time. It is becoming increasingly difficult for recipients to distinguish between serious and manipulated news, because not every widespread false report is easy to detect. There are grey areas between errors, deliberate falsifications of history, different perceptions and unconventional theses that have sometimes proved to be true. But even absurd conspiracy theories find their supporters as trust in the established media has fallen. On the other hand, oppositional opinions were also prematurely called conspiracy theories before their credibility could be verified. So they could already be exposed to absurdity without becoming dangerous to the dominant opinionwhich Marx's view represents the opinion of the ruling classes. Not only conspiracy theories, but also "fake news" are pejorative foreign attributions, while no one claims to represent such ones himself. Science is regarded as the most effective instrument against irrationality and lies. It has dealt extensively with media and conspiracy theories, but seems to lose both: its struggle for reason and its credibility in the accelerated age of social media. This article tries to find explanations for this development and asks about the perspectives of an credible communication.
\end{abstract}

Structured Abstract: The article deals with the circulation of so-called fake news and conspiracy theories. The relevance of this debate results from the political debates of recent years and the increase of misinformation. Last but not least, the social media are responsible for the fact that we are now experiencing a new form of communication which also facilitates the spreading of rumours. Due to the oversupply of information and digital communication, it is becoming more difficult for recipients to distinguish important from unimportant information. However, it is also becoming a challenge to filter between serious messages and invented or incorrect messages. The work asks why this is the case and what possibilities exist to prevent the abuse of media freedom. Specifically:

\footnotetext{
* Dr. Öğr. Üyesi, Necmettin Erbakan Üniversitesi, Güzel Sanatlar Fakültesi, Sinema-TV Bölümü Assistant Professor, Necmettin Erbakan University, Faculty of Fine Arts, Cinema-TV Department ORCID 0000-0002-4822-6899

zehra_ozkececi@yahoo.de

Cite as/ Atıf: Özkeçeci, Z. (2020). Zwischen Virtueller Realität und Alternativen Fakten Fake News und Verschwörungstheorien in den Sozialen Medien. Turkish Studies, 15(3), 2017-2031. https://dx.doi.org/10.29228/TurkishStudies.42696

Received/Geliş: 29 March/Mart 2020

Accepted/Kabul: 20 June/Haziran 2020

Copyright $($ ) MDE, Turkey

Checked by plagiarism software

Published/Yayın: 25 June/Haziran 2020

CC BY-NC 4.0
} 
Question 1) What is meant by fake news and conspiracy theories, what is the difference between them?

Question 2) Why are they currently booming?

Question 3) What counter-strategies are available for science?

In addition to conventional media theories, at least a rudimentary reference is made here to constructivism, which is based on the impossibility of recognising reality or of being able to gain more than a partial knowledge. Methodically, the source research of primary and secondary sources is used.

Many experts see the reason for the increase of conspiracy theories in the uncertainty of many media users who have lost their trust in official reports. Politics also contributes to this, because although much is said about transparency, in reality it is avoided in all sectors. The credibility of established media has been shaken by several factors. On the one hand, it was the well-known misuse of serious leading media for political and military propaganda, such as the Iraq war in 2003; on the other hand, technological development and digitalisation: The Internet and digital media have permanently changed the hierarchy between sender and receiver. Today, every user can also distribute messages or write messages himself.

Some conspiracy theories are completely absurd, others sound realistic and are therefore harder to refute. This is precisely why people believe in the simplest explanatory patterns; they often convey enemy images and make scapegoats responsible for social problems. So there are also theories that represent a danger or spread racist ideas. One example are terrorist attacks that occurred in reaction to false reports. Throughout history it has often been conspiracy theories that have led to pogroms or persecutions.

In addition to the earlier antisemitic conspiracy theories, today it is Islamophobia in particular that is being promoted, not only in the social, but also in the established mainstream media and politics. The example shows that conspiracy theories are not always a minority opinion, but that common mainstream theories should also be tested for their truthfulness.

However, the article also deals with the opposite phenomenon. Sometimes actual events are mistaken for conspiracy theories, while rumours and defamations are easier to enforce and difficult to refute. The work also asks about the scientific research of fake news and conspiracy theories. Due to the complex background, an interdisciplinary approach is recommended, because otherwise important aspects would be neglected. For example, psychology also plays an important role in the dissemination of fake news, not only political science or communication science issues.

The article also eamines the interfaces between reality and fiction, as conspiracy theories usually work with half-truths. In other words, this means that those who spread these theories believe it themselves. This also makes a difference between these theories and false reports that are spread as deliberate lies. Because reality can always only be partially recognized and is often reproduced in a distorted way even in high-quality media, it is difficult to distinguish between news with a real news value and those without a serious basis. Since even representatives of established politics such as the US President are spreading theses that were once considered to be conspiracy theories, the grey area between fake news, ideology and political propaganda has increased. Science can therefore only examine an object or a message with the observance of proven methods (verification, falsification, etc.) without being able to guarantee their truthfulness.

In the social media, algorithms often create filter bubbles by linking like-minded conspiracy theorists, who therefore see their views confirmed. In the resulting so-called echo chambers, information that disagrees with one's own opinion has no chance.

Various providers on the net and also Mark Zuckerberg have announced to take action against fake news and conspiracy theories. This is presented as a mean to prevent the widespread misuse of the media. On the other hand, this could also lead to censorship of democratic politics, where only the official version may be distributed. Data protectionists have protested against this and see their worst fears of a surveillance state confirmed. As this article also states, it is precisely the questioning of existing dogmas that is essential in order to find new insights. What someone may or may not believe is the private opinion of the believers, provided that this doesn't restrict the opinion or life of others. The article ends by pointing out that one's own reflection and critical ability is the best protection against being fooled by false reports or conspiracy theories.

Keywords: Media, Conspiracy theories, Fake News, Social Media, Science 
Abstract: Dieser Artikel setzt sich mit der Verbreitung von Fake News und Verschwörungstheorien im digitalen Zeitalter auseinander. Die sozialen Medien erleichtern es, jede realistische wie absurde Theorie ohne großen finanziellen Aufwand und ohne akademische Voraussetzungen weltweit zu publizieren. Obwohl es in der Geschichte immer schon erfolgreiche Versuche gab, Falschmeldungen und Gerüchte zu verbreiten, ermöglichen es heute die technologischen Fortschritte, ein ungleich breiteres Publikum in Echtzeit zu erreichen. Für die RezipientInnen wird es zunehmend schwieriger, zwischen seriösen und manipulierten Nachrichten zu unterscheiden, denn nicht jede verbreitete Falschmeldung ist leicht als solche zu erkennen. Es bestehen Grauzonen zwischen Irrtümern, absichtlichen Geschichtsfälschungen, unterschiedlichen Wahrnehmungen und unkonventionellen Thesen, die sich manchmal doch als wahr herausgestellt haben. Doch selbst absurde Verschwörungstheorien finden ihre AnhängerInnen, da das Vertrauen in die etablierten Medien gesunken ist. Umgekehrt wurden aber auch unhinterfragt oppositionelle Meinungen voreilig als Verschwörungstheorien bezeichnet, bevor ihre Glaubwürdigkeit überprüft werden konnte. So konnten sie bereits der Lächerlichkeit preisgegeben werden, ohne der herrschenden Meinung - die laut Marx die Meinung der Herrschenden repräsentiert - gefährlich zu werden. Denn sowohl „Verschwörungstheorien“ wie auch „Fake News" sind abwertende Fremdzuschreibungen, während keiner für sich beansprucht, solche selbst zu vertreten. Die Wissenschaft gilt als wirksamstes Mittel gegen Irrationalität und Lüge. Sie hat sich umfassend mit Medien und Verschwörungstheorien auseinandergesetzt, aber scheint im beschleunigten Zeitalter der sozialen Medien sowohl ihren Kampf um die Vernunft als auch ihre Glaubwürdigkeit zu verlieren. Dieser Artikel sucht nach Erklärungen für diese Entwicklung und fragt nach den Perspektiven einer glaubwürdigen Kommunikation.

Schlüsselwörter: Medien, Verschwörungstheorien, Falschnachrichten, Soziale Medien, Wissenschaft

\section{Einleitung}

Es war ausgerechnet der amerikanische Präsident Donald Trump und seine Administration, die den Begriff der Fake-news wie auch der ,alternativen Fakten“ populär machten, während sich Trump selbst nicht unwesentlich an deren Verbreitung beteiligte. Seine täglichen Botschaften auf Twitter sind legendär. Mit „Fake-news“ waren aber nicht abgehobene Verschwörungstheorien, sondern die alltäglichen Nachrichten der bürgerlichen Medien gemeint. Dadurch wurde der Fokus darauf gerichtet, wie viel eigentlich von der Berichterstattung, die uns als rational und seriös erscheint, noch geglaubt werden kann. 2014 wurde der Begriff „Lügenpresse“ in Deutschland zum Unwort des Jahres erklärt: begründet wurde das mit der Verwendung des Begriffs im Ersten Weltkrieg, aber auch von den Nationalsozialisten gegen eine oppositionelle bzw. ausländische Presse. Mittlerweile wird der Terminus von der antiislamischen Bewegung Pegida eingesetzt (cf. APA/Reuters 2015), was ihn ebenfalls für eine seriöse Medienkritik disqualifiziert. Trotzdem haben die Presse und die Medien an Glaubwürdigkeit verloren, während die Medien selbst versuchen, die Infragestellung der Mainstream-Berichterstattung nur rechten oder sogar rechtsradikalen Strömungen zuzuschreiben. In diesem Artikel werden Antworten auf die Frage gesucht, warum im Zeitalter der sozialen Medien Verschwörungstheorien und Fake-News Hochkonjunktur haben. Was wird überhaupt darunter verstanden und gibt es überhaupt erfolgreiche Gegenstrategien?

\section{Zur Problemstellung}

Eng verwandt mit den Fake-News sind die Verschwörungstheorien, die trotzdem nicht einfach mit ihnen gleichgesetzt werden können. Falschmeldungen können als absichtliche Lügen bezeichnet werden, was bei Verschwörungstheorien nicht automatisch der Fall sein muss. Oft werden sie selbst von ihren UrheberInnen geglaubt, bevor sie über alternative und vor allem über die sozialen Medien verbreitet werden. Auch ist nicht gänzlich auszuschließen, dass Verschwörungstheorien von der Realität bestätigt werden. Der Definition nach handelt es sich eben einfach um Theorien oder „,Vermutungen “ über Verschwörungen, die in der Geschichte ja tatsächlich gehäuft auftreten und nicht nur erfunden wurden.

„Eine Verschwörungstheorie ist monokausal. Das bedeutet, dass bestimmte soziale Phänomene oder historische Ereignisse auf Verschwörungen zurückgeführt werden. Erklärungen 
werden dadurch vereinfacht. Hinter allen Entwicklungen der Welt stecke ein geheimer Plan: Nichts geschieht zufällig und alles scheint irgendwie miteinander verbunden zu sein. " (Landeszentrale für politische Bildung 2019)

Die Landeszentrale ignoriert hier politische Strategien, die tatsächlich unter Ausschluss der Öffentlichkeit stattfinden. Auch bedeutet die Ansicht, dass ,alles miteinander verbunden“ "bzw. in Beziehung steht, noch keine Verschwörungstheorie, sondern eine ganzheitliche Sicht auf die Welt. Diese Sichtweise widerspricht zwar der westlichen Praxis, alles zu selektieren und isoliert zu betrachten, hat aber die Wissenschaft auf ihrer Seite, während Segmentierung und Segregation eher mit dem Vorwurf des Eurozentrismus konfrontiert werden (cf. Khella 1995, 13). Die Definition von Verschwörungstheorien gestaltet sich also schwierig, denn es gibt keine einheitliche wissenschaftliche Kategorisierung, wie auch die Landeszentrale für politische Bildung feststellt (Landeszentrale für politische Bildung 2019).

Verschwörungstheorien bestehen seit Jahrtausenden und korrelieren mit unseren Vorurteilen und Feindbildern. Doch durch das Internet und die sozialen Medien entstanden neue Möglichkeiten, um Fake-News zu verbreiten. Die erhoffte Demokratisierung, die aufgrund der neuen Medien prognostiziert wurde, fand insofern nur bedingt statt.

„Verschwörungstheorien zirkulieren durch technologische Neuerungen schneller denn je. Bei Google-Suchen und auf Facebook wird man zu Themen wie 9/11 schnell mit ihnen konfrontiert, Bots verbreiten sie automatisiert. “ (de la Riva 2019)

Doch es gibt noch eine andere Beziehung zwischen Fake-News und Verschwörungstheorien. Gerade die Angst vor Lügen in den Mainstreammedien oder die Wut, die RezipientInnen darüber empfinden, macht jene Medien interessant und glaubwürdiger, die eine gänzlich andere Sicht vertreten. Tatsache ist, dass die wichtigsten Medienkartelle dieser Welt mit politischer und ökonomischer Macht verbunden sind, weshalb ihnen von großen Teilen der Bevölkerung misstraut wird. (cf. Krammer 2015, 27-32) Wenn aber die offizielle Version als Fake betrachtet wird, erhalten alternative Versionen einer Nachricht und selbst ausgefallene Narrative eine realistischere Aura.

Der Kulturanthropologe Didier Fassin geht davon aus, dass Verschwörungstheorien viel über tatsächliche Ungerechtigkeiten in der Gesellschaft aussagen, da es in ihnen meistens um Ungleichheiten geht, die zwar erfahren, aber nicht wirklich durchschaut werden (cf. Riga 2019). Viele Menschen wissen, dass ihnen nicht alles berichtet wird, was sie wissen möchten, und dass die vielzitierte Transparenz der politischen Parteien oft nur eine Phrase ist. Gleichzeitig spricht Fassin auch das Problem der Überinformation aus, die es erschwert, zwischen wichtigen und unwichtigen oder auch zwischen wahren und erfundenen Informationen zu filtern. Und er sagt:

"Je mehr wir wissen, desto deutlicher wird, wie viel wir nicht verstehen..." (de la Riva 2019).

\section{Formen von Verschwörungstheorien}

Es gibt Youtubevideos über die absurdesten Verschwörungstheorien, die aber manchmal trotzdem oder gerade deswegen von Millionen angeklickt werden.

Es gibt Theorien, dass die Erde eine Scheibe ist oder zumindest hohl und darin ganze Zivilisationen leben. Es gibt die Vorstellung von Reptiloiden und Dämonen, es gibt Geisterjäger und Prominente, die sich okkulten Sekten angeschlossen haben. Der Klimawandel sei eine Erfindung und die Mächtigen dieses Planeten seien in Wirklichkeit Außerirdische oder in Geheimbünden organisiert. ExpertInnen sehen darunter eine Möglichkeit, in einer komplizierten Welt einen Halt zu finden oder sich selbst eine größere Bedeutung zu verleihen (cf. Landeszentrale für politische Bildung 2019). Doch sie warnen gleichzeitig vor einem bedrohlichen Potential:

„Verschwörungstheorien sind also nicht nur abstrus-kreative Gedankenspiele von kreativen Spinnern, sondern können auch Gefahren darstellen: Sie können antisemitisches und 
antipluralistisches Denken aufweisen und führen so zu einer Radikalisierung. “ (Landeszentrale für politische Bildung 2019)

Andere Theorien werden als Verschwörungstheorien bezeichnet, obwohl ihr Wahrheitsgehalt keineswegs wissenschaftlich ausgeschlossen werden kann, sondern nur von der offiziellen Darstellung abweicht. Oft werden auch einfach oppositionelle Meinungen als Verschwörungstheorien lächerlich gemacht. Es gibt den ironischen Spruch: „Nur weil du paranoid bist, heißt das nicht, dass du nicht verfolgt wirst." Die als Witz verstandene Phrase trägt einen wahren Kern. Viele fühlen sich überwacht oder dass sie ins Visier von Geheimdiensten oder Regierungen geraten sind - oft ein psychologisches Problem, aber wie spätestens seit Edward Snowden bekannt geworden ist, kann es sich dabei auch um eine tatsächliche Gefahr handeln (Ibid.)

Das Internet hat die Möglichkeiten der Nachrichtenweitergabe natürlich immens erweitert und auch die Verbreitung von Verschwörungstheorien erleichtert. Jede obskure Theorie kann mit wenigen Klicks im World Wide Web geteilt werden. Auf den Foren, auf Youtube und anderen sozialen Medien wie Facebook, Twitter usw. tauschen sich die User über ihre Ansichten aus und fühlen sich von den anderen bestätigt. Doch nicht nur die Gefahren des Missbrauchs sozialer Medien, sondern auch die Partizipationsmöglichkeiten und das Kommunikationsspektrum wurden dadurch erweitert. Und nicht nur die Postings in den Foren, auch die Nachrichten in den bürgerlichen Leitmedien sollten nicht einfach unhinterfragt übernommen werden, auch wenn der etablierte Journalismus den Zeiten nachtrauert, wo die Hierarchie zwischen Sender und Empfänger noch eindeutiger getrennt war.

Tatsache ist, dass alle Regierungen Milliarden für Geheimdienste und Spionage ausgeben, auch für Desinformation und Propaganda (cf. Krammer 2015, 34). In manchen Staaten arbeiten zehntausende Menschen für diese geheimen Apparate. Das befördert die Phantasie über vermeintliche oder tatsächliche Absichten, die dahinter stehen könnten und schafft Nahrung für neue Verschwörungstheorien. Je mehr vor den Bürgern eines Landes verborgen wird, umso stärker verbreiten sich Gerüchte und das Gefühl, von einer unsichtbaren Macht bedroht zu werden. Dass den Menschen wichtige Informationen verschwiegen bzw. vorenthalten werden, wird auch von de la Riva bestätigt und er sieht in der dadurch entstehenden Verunsicherung die eigentliche Grundlage für Verschwörungstheorien (de la Riva 2019).

Populistische Parteien können darauf aufbauen und bestimmte Sündenböcke für politische und gesellschaftliche Missstände verantwortlich machen. Hier wird nicht nur mit Vorurteilen, sondern auch mit Halbwahrheiten gearbeitet - bei ihnen handelt es sich nicht um die halben, sondern um die größeren, weil besser versteckten und deshalb effektiveren Lügen. Deshalb werden gerade auf rechten Netzwerken rassistische Theorien verbreitet, die mit Flüchtlings- und Migrationsströmen zusammenhängen. In der Geschichte wurden auch viele antisemitische Verschwörungstheorien ausgetauscht, die nicht unwesentlich zum Holocaust und zur Ermordung von Millionen jüdischer Menschen beigetragen haben (Landeszentrale für politische Bildung 2019).

Heute ist es deshalb in vielen westlichen Ländern und insbesondere in den Nazinachfolgestaaten Deutschland und Österreich tabu, den immer noch vorhandenen Antisemitismus offen zu zeigen, der sich oft hinter einer bedingungslosen Israelsolidarität versteckt. Dafür kann die Islamfeindlichkeit offen gezeigt und als Religionskritik verharmlost werden. Viele stereotypische Zuschreibungen wurden dabei vom klassischen Antisemitismus übernommen und werden heute auf Muslime projiziert. Zu den gängigsten Verschwörungstheorien zählen heute die „Islamisierung des Abendlandes“ und die höhere Kriminalitätsrate von MigrantInnen aus islamischen Ländern. Auf den Zusammenhang von Antisemitismus und Islamfeindlichkeit weisen Islamophobiker unfreiwillig hin, wenn sie heute in einer von offiziellen Kreisen anerkannten These behaupten, der Antisemitismus werde aus islamischen Ländern importiert. Nun wird jeder seriöse Antisemitismusforscher dieser Verschwörungstheorie entschieden widersprechen und auf den 
europäischen Ursprung des rassistischen Antisemitismus verweisen. Doch hat sich diese Theorie mittlerweile im Mainstream etabliert und wird nicht nur von rechten PolitikerInnen, sondern auch von wissenschaftlichen Institutionen vertreten (cf. Landeszentrale für politische Bildung 2019).

Anerkannte Islamwissenschafter oder- experten sind selten Muslime. In den offiziellen westlichen Medien wird auch kaum ein Experte eingeladen, der keine massive Kritik am Islam äußert. Islamkritiker und Islamexperte sind in westlichen Ländern sehr oft identisch. Deshalb grassieren nicht nur unzählige Verschwörungstheorien über Muslime, sondern es entstehen im Gegenzug auch zahlreiche Verschwörungstheorien in Teilen der muslimischen Communities selbst, um die ihnen entgegengebrachte Feindseligkeit zu erklären. Skeptisch gegenüber den offiziellen Medien, die permanent Gerüchte über sie verbreiten, werden Muslime leichter anfällig für „alternative Fakten“. Anders ausgedrückt erzeugen erst die Vorurteile gegen Muslime das, was sie eigentlich vorgeben zu bekämpfen. So entstehen manchmal tatsächlich antisemitische Thesen als Erklärungsmuster für islamophobe Strömungen. Der Antisemitismus wird also nicht von Muslimen importiert, sondern von europäischen IslamexpertInnen herbeigeredet und Muslimen untergeschoben. Doch keinesfalls ist dieser Antisemitismus mehrheitsfähig. Denn die islamischen Communities wissen über die „Doppelgestalt von Antisemitismus und Islamfeindlichkeit“ Bescheid und sehen in beiden Erscheinungsformen eine Variante des kulturellen Rassismus (Balibar 2006, 3439).

Eine der wichtigsten Gemeinsamkeiten zwischen Antisemitismus und Araberfeindlichkeit findet sich nach Balibar in den zugeschriebenen Verschwörungstheorien: „Das Szenario der Verschwörung. Hier knüpft der Komplex des Judenhasses und des Araberhasses am stärksten an. “ (Balibar 2006, 38) Wenn Etienne Balibar auf die Parallelen zwischen Antisemitismus und „Araberfeindlichkeit bzw. die Islamfeindlichkeit“, verweist, leugnet er nicht, dass Millionen arabischer Menschen anderen Konfessionen angehören, doch ändert das nichts an den ihnen entgegengebrachten Vorurteilen. Davon betroffen sind übrigens auch alle einer bestimmten Verschwörungstheorie zugeordneten Menschen, auch wenn es sich bei ihnen um Iraner, um Sikhs oder konvertierte EuropäerInnen handelt.

Das Beispiel zeigt, dass es sich bei Verschwörungstheorien nicht unbedingt um eine Minderheitenerscheinung in der Bevölkerung handeln muss, sondern dass sie häufig mit der Mehrheitsmeinung ident sind.

\section{Die wissenschaftliche Erforschung von Verschwörungstheorien}

Es gibt mehrere wissenschaftliche Forschungen über Verschwörungstheorien und auch universitäre Angebote zu diesem Thema. Verschwörungstheorien können nicht nur aus psychologischer, sondern auch aus kommunikationswissenschaftlicher, historischer, soziologischer oder politikwissenschaftlicher Perspektive betrachtet und untersucht werden. Auch medizinische Fragen spielen eine Rolle, weil sie entweder selbst im Fokus von Verschwörungstheorien stehen oder weil die Grauzone zwischen dem Verbreiten von subjektiven Wahrnehmungen und einer psychischen Erkrankung überschritten wurde. Obwohl es hier vor allem um die Verbreitungsmöglichkeiten über die sozialen Medien geht und deshalb die publizistische Herangehensweise interessiert, können bei diesem Thema die anderen Aspekte und Zugänge nicht ignoriert werden. Verschwörungstheorien können eigentlich nur interdisziplinär untersucht werden, weil ansonsten das breite Spektrum von Einflüssen vernachlässigt wird,

Die Organisatoren eines Q-Tutoriums an der Berliner Humboldt-Universität über Verschwörungstheorien berichten über die Hindernisse eines wissenschaftlichen Zugangs, die mit diesem Thema verbunden sind. Sie wiesen darauf hin, dass sich zuerst viele Studierende zum Tutorium meldeten, weil Verschwörungstheorien eine große Faszination auslösen, dass es aber schwierig wurde, sobald es um die Entwicklung eines seriösen Forschungsdesigns und das Herausarbeiten der Forschungsfragen ging (cf. Buchmayr/ Köhler 2016/2017, 3). 
Schon die Definition gestaltet sich schwierig. Wie der Name bereits sagt, sind Verschwörungstheorien mit der Vorstellung verbunden, dass gewisse Phänomene, Ereignisse bzw. Entwicklungen das Ergebnis einer Verschwörung oder eines geheimen Plans sind. Theorien über Verschwörungen werden allgemein abwertend gebraucht, wobei darunter meistens eine Verabredung zu geheimen, weil kriminellen Handlungen verstanden wird, von der ein größerer Teil der Menschheit betroffen sei. Mittlerweile werden aber auch alle von der offiziellen Version abweichenden Theorien als Verschwörungstheorie bezeichnet, auch wenn sie jetzt nichts mit einer klassischen Verschwörung zu tun haben. Was sich irgendwie verrückt anhört wie die Anwesenheit von Aliens hat höchstens insofern mit einer Verschwörungstheorie zu tun, als den Regierungen unterstellt wird, darüber Bescheid zu wissen und es der Bevölkerung zu verheimlichen.

Der Unterschied zwischen einer tatsächlicher Verschwörung und der Theorie ist nicht immer leicht zu erkennen, denn die erfolgreichsten Verschwörungstheorien basieren nicht auf bloßen Phantasien, sondern auf einem realen Hintergrund (cf. Wieselberg 2019): zum Beispiel wurden Vereinigungen wie die Bilderberger, die Freimaurer und sogar die Illuminaten oder die Templer ja nicht einfach frei erfunden, sondern existierten tatsächlich oder treffen sich noch bis zum heutigen Tag. Die Verschwörungstheorie entsteht aufgrund einem Konstrukt, das auf der Basis dieses „realen Hintergrunds" geschaffen wurde - sie baut etwa auf geheimen Treffen von Freimaurerlogen auf und interpretiert, was dort besprochen werden könnte. Oder viele Anhänger grenzen sich von anderen VerschwörungstheoretikerInnen $\mathrm{ab}$, indem sie sich auf anerkannte Wissenschaftler oder etablierte Theorien berufen. Vor allem Einstein und Tesla werden immer wieder bemüht, um bestimmte Ansichten zu verteidigen, so dass weniger belesene RezipientInnen den Eindruck gewinnen könnten, die wissenschaftlichen Koryphäen hätten ähnliche Verschwörungstheorien vertreten. Deshalb ist ein verbreiteter Vorwurf, der gegen Verschwörungstheoretiker erhoben wird, pseudowissenschaftlich vorzugehen (cf. Landeszentrale für politische Bildung 2019).

Die Realität kann nur in beschränktem Ausmaß erkannt und davon wiederum nur ein Ausschnitt kommuniziert werden. Wer selbst einmal zum Gegenstand in der Berichterstattung wurde und sei es nur in einem harmlosen Beitrag in einer regionalen Zeitschrift, weiß, dass es selten eine wirklich authentische Meldung gibt, die frei von Verzerrungen wäre. Das passiert nicht, weil sämtliche JournalistInnen den Auftrag haben, mit Falschmeldungen eine Form von Gehirnwäsche zu betreiben, was schon wieder eine andere Verschwörungstheorie wäre. Tatsächlich besteht aber nur eine begrenzte Wahrnehmung, die von der eigenen Realität beeinflusst wird. Die Wirklichkeit kann nie vollständig erfasst werden, wie es der Konstruktivismus seit jeher feststellte. Der Konstruktivismus leugnet den Anspruch auf Wahrheit oder gar absolute Wahrheit und geht davon aus,

„, dass die Aussagen über die Wirklichkeit dem eigenen Erleben, der eigenen Geschichte, der eigenen Entwicklung und den eigenen (beschränkten) physischen Möglichkeiten der Wahrnehmung entspringen. Aufgabe des Konstruktivismus ist es deshalb zu zeigen, wie Wirklichkeitskonstruktionen gemacht werden. Mit anderen Worten: Der Konstruktivismus nimmt Abschied von der absoluten Wahrheit. " (Feess/Thomas 2019)

Deshalb kann die Wirklichkeit nicht gänzlich erkannt werden, es gibt nur eine Annäherung an sie. Dieser Umstand erschwert es der Wissenschaft, Gerüchten mit Fakten zu begegnen. „Wissenschaftlich erwiesen“ ist kein Dogma. Es bedeutet immer nur, sich auf den aktuellen Stand der Forschung, der ständig überprüft und bei Bedarf falsifiziert wird, zu berufen. Genau diese Praxis unterscheidet eine wissenschaftliche Herangehensweise von einer Verschwörungstheorie.

\section{Verschwörungstheorien von oben}

Viele Verschwörungstheorien sind in den gesellschaftlichen Eliten angekommen und werden auch von Regierungsmitgliedern vertreten. Die US-Regierung unter Trump teilt und verbreitet mehrere bekannte davon, darunter den Vorwurf von der „Klimalüge“, also die Leugnung des durch 
den Menschen verursachten Klimawandels. Viele Verschwörungstheorien orientieren sich am Gegensatz zwischen Elite und Volk (cf. de la Riva 2019). Verbunden ist diese Tendenz mit der Anschauung, dass Eliten durch Bildung, nicht durch Macht oder Vermögen definiert werden. Als US-Präsident und Milliardär wäre Trump zumindest der Definition nach der mächtigste Mann der Welt, doch weil er sich nicht wie ein Intellektueller, sondern unhöflich und oft aggressiv äußert und sich politisch nicht an diplomatische Gepflogenheiten hält, gilt er nicht als Teil der Elite (cf. Özkececi 2018, 29).

In seiner Administration werden gleich mehrere Verschwörungstheorien vertreten, was auch mit der dogmatisch evangelikalen Ausrichtung einiger Abgeordneter zu tun hat (cf. Schwarz 2016). Meistens steht die politische Opposition im Focus dieser Behauptungen, die aktuell natürlich primär über soziale Medien verbreitet werden.

\section{Die Gefahren von Verschwörungstheorien}

Indessen zeigte sich erst vor kurzem, wie Verschwörungstheorien auch zu Gewaltanwendung führen können. Ein Bewaffneter stürmte 2016 eine Pizzeria in Washington, weil im US-Wahlkampf behauptet worden war, dass von dort ein Kinderpornoring geleitet würde. Auch die demokratische Präsidentschaftskandidatin Hillary Clinton wurde beschuldigt, an dieser kriminellen Organisation beteiligt zu sein. Selbst der Trump-Berater Michael Flynn hatte entsprechende Nachrichten über die sozialen Medien verbreitet (cf. Schwarz 2016). Als unmittelbare Folge dieser Verschwörungstheorien erhielten die betreffende Pizzeria wie auch andere angeblich involvierte Restaurants Hassmails und Drohungen. Im Dezember 2016 stürmte ein Bewaffneter die Pizzeria, um die laut der Verschwörungstheorie festgehaltenen Kinder zu befreien (ibid.).

Von rechtsradikalen Verschwörungstheorien beeinflusst, stürmte ein Neofaschist zwei Moscheen in Christchurch (in Neuseeland) und richtete ein Blutbad an betenden Gläubigen an. 51 Menschen wurden bei diesem Amoklauf ermordet. Der Attentäter von Christchurch hatte seinen Anschlag online gestellt, um über die sozialen Medien seine Verbrechen publik zu machen (cf. Zeitonline 2019). Davon inspiriert plante ein Trittbrettfahrer eine Synagoge in Halle zu überfallen und ein ähnliches Massaker anzurichten. Da er an der Eingangstüre der Synagoge scheiterte, tötete er eine Passantin und den Kunden eines Dönerrestaurants, den er für einen Ausländer hielt. Nach einem Nature Artikel (Johnson et al, 2019) gäbe es eine epidemische Zunahme von Hassverbrechen und Selbstmorden, die mit ihrer digitalen Verbreitung in einen Zusammenhang gestellt werden können. Das beginnt bei den Hass- und Drohmails und endet wie beim Christchurchattentäter bei der in Echtzeit übertragenen Zurschaustellung eines Massakers. Johnson spricht von Hate-Clustern, die mit speziellen mathematischen Computerprogrammen bekämpft werden müssten, während die individuelle Bekämpfung einzelner Hassposter wenig effektiv sei (ibid.). Bei genauerer Betrachtung dieser „Hate-Cluster“ fällt auf, dass beinahe jedes dieser Hassverbrechen, das mit Hilfe der sozialen Medien geteilt oder auch begangen wird, ihren Ursprung in Verschwörungstheorien hat, wobei die meisten VT erst über und durch soziale Medien entstehen und verbreitet werden konnten, wie die Beispiele von Christchurch und Halle deutlich zeigen.

\section{Die Grauzone}

Das Problem im Kampf gegen Verschwörungstheorien ist die Grauzone zwischen tatsächlichen oder vermeintlichen Fake-News, zwischen persönlichen Einschätzungen und offiziellen Verlautbarungen oder Unterstellungen. Auch sind die meisten Verschwörungstheorien nicht unrealistischer als die Glaubensinhalte anerkannter Religionsgemeinschaften und fallen unter die persönliche Meinungsfreiheit. Nicht alle, die der offiziellen Darstellung des 11. Septembers 2001 und den Angriffen auf das World Trade Center und das Pentagon skeptisch gegenüberstehen, können schon als VerschwörungstheoretikerInnen bezeichnet werden, obwohl das von einigen „Verschwörungstheorie- Theoretikern“ manchmal so gesehen wird. Auch von offizieller Stelle wurde dem Zweifel an der Mainstreamversion so viel Platz eingeräumt, dass eine 
Untersuchungskommission eingesetzt und ein umfassender und nach Kritik überarbeiteter Report über deren Ergebnisse veröffentlicht wurde (The 9/11 Commission Report, 2004). Tatsächlich wurde auch in diesem Report eine Verschwörungstheorie verbreitet, die davon ausgeht, dass sich Islamisten „verschworen“ haben, um diesen verheerenden Anschlag zu begehen - das gilt zwar als offizielle und deshalb „wahre“ Theorie, die auch von der 9/11 Kommission bestätigt wurde, aber es ist trotzdem unzweifelhaft eine „Verschwörungs“"-theorie.

Selbst wer absurde Theorien verbreitet, muss nicht unbedingt unter Wahnvorstellungen leiden: meistens liegt das, was wir glauben, an den Informationen, die wir erhalten (cf. Muth 2018). Ein Großteil dieser Informationen wird über Bildungseinrichtungen und die Medien vermittelt. Wer absurde Theorien vertritt, hat in der Regel nur unzuverlässigen Kommunikationsmitteln vertraut.

Auch die klügsten Menschen bauen ihre Theorien auf dem Vorwissen auf, das andere vor ihnen erschlossen haben und denen vertraut wird. Dieses Vertrauen wird oft vorschnell entgegengebracht und viele wissenschaftliche Dogmen werden später als Irrtümer entlarvt. Fast 100 Jahre lang wurde der angeblich hohe Eisengehalt im Spinat nicht in Frage gestellt, bis kritische Geister feststellten, dass sich die Urheber dieses Dogmas um eine Kommastelle geirrt hatten (cf. Gesundheit.de, 2017). Generationen von Kindern wurden gezwungen, Spinat zu essen, Werbung und Ernährungsberater beharrten auf den ursprünglichen Zahlen und selbst die Popeye-Cartoons basierten auf diesen irrtümlichen Annahmen. Das Beispiel zeigt, dass der Mainstream nicht seriöser sein muss als die oft absurden Thesen von Einzelpersonen. Tatsächlich wurden die meisten wissenschaftlichen Erkenntnisse von individuellen ExpertInnen erst gegen den Widerstand der vorherrschenden Meinung entwickelt.

Umgekehrt bewirkt aber eine vorschnelle Gleichsetzung aller „originellen“ Ideen mit Verschwörungstheorien, dass sich möglicherweise bereichernde Innovationen nicht durchsetzen können. Wenn skurrile Theorien gemeinsam mit durchaus realistischen, aber unkonventionellen oder abweichenden Meinungen auf eine Stufe gestellt werden, findet eher eine Forderung nach Konformität statt als die Widerlegung von Verschwörungstheorien. Der mündige Bürger, der nicht jede Meldung kritiklos hinnimmt, wird genauso ins Lager der Verschwörungstheoretiker verbannt wie der mündige Patient, der nicht einfach Medikamente schluckt und vor einer Impfung wissen möchte, was in seinen Körper injiziert wird.

„Fast die Hälfte der Deutschen glaubt, dass Politiker nur Marionetten anderer Mächte sind. ...So meinen beispielsweise 46 Prozent der Befragten, es gäbe geheime Organisationen, die Einfluss auf politische Entscheidungen haben. Fast ein Viertel der Befragten meint, Medien und Politik steckten unter einer Decke, und jede zweite befragte Person gibt an, den eigenen Gefühlen mehr zu vertrauen als Expertinnen oder Experten." (Landeszentrale für politische Bildung 2019)

Die deutsche Landeszentrale für politische Bildung sieht in dieser Entwicklung eine Gefahr. Dabei könnte es auch als Stärke betrachtet werden, wenn Menschen ihre Autoritäten hinterfragen. Gerade der/die kritische MedienkonsumentIn wird gegenüber Verschwörungstheorien und Fakenews weniger anfällig sein als RezipientInnen, die alles glauben, was aus offiziellen Kreisen vermittelt wird.

Es könnte auch gesagt werden, dass Verschwörungstheorien oft als solche bezeichnet werden, wenn sie den Ideologien und der politischen Ausrichtung des Mainstreams widersprechen. Andere Theorien, die genauso wenig bewiesen und genauso von Verschwörungen ausgehen, gelten als seriös und haben ein wesentlich besseres Image. Sie haben sogar Nachrichtenwert und werden von den offiziellen Medien verbreitet: etwa dass der US-Wahlkampf von Russland organisiert worden sei oder dass russische Oppositionelle im Ausland im Auftrag der russischen Regierung ermordet werden, gilt schnell als bewiesen, während die Behauptung, der US-Geheimdienst stecke hinter politischen Umstürzen, leicht als Verschwörungstheorie abgewertet wird, obwohl geöffnete CIA-Archive genau das bestätigten (cf. CIA 2019a). 
Historisch betrachtet müsste man Marx widersprechen, der die Geschichte als „Geschichte von Klassenkämpfen“ definierte und sie durch die „Geschichte von Verschwörungstheorien, Fakenews und Missverständnissen" ersetzen. Ob es die angebliche christliche Brandstiftung von Rom zu Zeiten Neros betrifft oder den angeblichen Gottesbefehl im Alten Testament, andere Völker oder Stämme von dem Boden zu vertreiben, auf dem man selbst gerne leben möchte. Bekannte Verschwörungstheorien waren auch die Fälschung der „Protokolle der Weisen von Zion“, die inszenierten Angriffe Polens auf Nazideutschland (,Ab heute, 5.Uhr 45 wird zurückgeschossen!“), um den Krieg gegen Polen und damit den Beginn des 2. Weltkriegs zu legitimieren, bis zu den Massenvernichtungswaffen im Irak.

Tatsächlich besteht eine Grauzone zwischen politischer Propaganda, Ideologie und Verschwörungstheorien. Es gibt kaum einen Krieg, der nicht mit Hilfe von Fake-News und Verschwörungstheorien geführt worden wäre. Sowohl der Irakkrieg 1991 als auch die Intervention von 2003 basierten auf Medienlügen. Die NATO-Intervention gegen Jugoslawien 1999 gründete auf einer klassischen Verschwörungstheorie, dem sogenannten Hufeisenplan: die serbische Verschwörung für ethnische Säuberungen, die sich schnell als Fälschung herausstellte (Hofbauer 2001,114).

Eine Verschwörungstheorie gilt nur solange als Verschwörungstheorie, bis sie durch glaubhafte Beweise bestätigt wird. Es gibt gar nicht so wenige Fälle, wo sich vermeintliche Verschwörungstheorien als wahr herausstellten, während umgekehrt viele offizielle Versionen als Medienlügen entlarvt wurden (cf. Muth 2018).

Da die CIA seit 2009 zur Publikation von Dokumenten verpflichtet ist, die 25 Jahre oder älter sind (Executive order 13526), haben sich tatsächlich viele früher als Verschwörungstheorien bezeichnete Gerüchte bestätigt. Interessanterweise wird etwa darin zugegeben, dass die CIA jahrzehntelang über UFO-Sichtungen forschte (CIA 2017, 1967) und auch darüber berichtete, dass es darüber Forschungen in China gab (CIA 1990). Auch stellten sich viele Vorwürfe gegen den amerikanischen Geheimdienst als wahr heraus - etwa die Unterstützung des Umsturzes im Iran und die Absetzung des gewählten Präsidenten Mossadegh oder die Förderung der Putschisten in Chile und anderen süd- und mittelamerikanischen Staaten. Whistleblower wie Edward Snowden berichteten darüber, wie NSA-Agenten die ganze Welt bespitzelten (cf. Muth 2018). Auch Menschenversuche mit ,chemischen, biologischen und radiologischen Substanzen“ zur Bewusstseinskontrolle - wie das lange belächelte MK-Ultra Programm - wurden tatsächlich durch die CIA bestätigt (CIA 2019 b).

\section{Die Reaktion der Sozialen Medien}

In den sozialen Medien entstehen Filterblasen, weil Algorithmen die Suchgewohnheiten von Usern speichern und die gesendeten Informationen danach gefiltert werden (Landeszentrale für politische Bildung 2019). So werden in den sozialen Medien Verschwörungstheoretiker eher mit anderen Verschwörungstheoretikern vernetzt und finden zusätzlich in den Suchmaschinen zuerst jene Vorschläge, die ihren Interessen entsprechen. Damit entstehen sogenannte Echokammern, wo nur mehr Gleichgesinnte miteinander kommunizieren und andere Meinungen gar nicht mehr vermittelt werden. Natürlich fördert diese Entwicklung die Entstehung wie auch die Bestätigung von Verschwörungstheorien, da andere Sichtweisen vernachlässigt und kaum noch in Betracht gezogen werden (Ibid.). Eine Konfrontation mit Fakten, die der eigenen Weltanschauung nicht entsprechen, wird dadurch von vornherein ausgeschlossen (cf. Wieselberg 2019). Deshalb werden Anti-HassCluster empfohlen, in denen Andersdenkende miteinander diskutieren können. Dieser Austausch könnte die Entstehung von Dogmenbildung verhindern (cf. Wieselberg 2019).

Mark Zuckerberg hat in der nachträglichen Auseinandersetzung über den US-Wahlkampf 2016 versprochen, auf Facebook Schritte gegen Fake-News zu setzen (Huber 2016). 
"Das wichtigste, das wir tun können, ist, unsere Fähigkeiten zu verbessern, Fehlinformationen als solche zu erkennen. Das heißt, dass wir bessere technische Systeme brauchen, die Inhalte schon als falsch erkennen, bevor sie Nutzer als Falschmeldung markieren." (Ibid.)

Insbesondere nachdem immer mehr Nutzer gesperrt worden waren, weil ihnen durch Algorithmen bescheinigt worden war, Hassverbrechen im Netz zu begehen, kam es zu Diskussionen über die Legitimität dieser elektronischen Systeme. Datenschützer befürchten einen fahrlässigen Umgang mit Informationen und sprechen von Zensur.

So wie früher die menschlichen Forenwächter sind auch die digitalen Filtersysteme nicht frei von subjektiven Einschätzungen der Menschen, die sie programmiert haben. Es ist nur noch schwieriger geworden, mit ihnen zu diskutieren.

Gegen Wikipedia gab es 2019 ein Gerichtsurteil, das die Deanonymisierung von AutorInnen erlaubt, nachdem eine bestimmte ideologisch gefärbte Gruppierung die Artikel auf der Medienplattform in ihrem Sinn beeinflusst hatte (Schmid/ Muzayen, 2019). Obwohl auch in diesem konkreten Fall die Medien, die Namen von Autoren veröffentlicht hatten, als Verschwörungstheoretiker bezeichnet wurden (Ibid.), zeigte der Prozess, dass es sich bei den veröffentlichten Artikeln auf Wikipedia um keine wertfreien bzw. objektiven Beiträge handelt, sondern dass sie die politische Orientierung der AutorInnen spiegeln.

\section{Fazit}

Alle Experten beobachten eine Zunahme von Verschwörungstheorien und Fake-News, die insbesondere über die sozialen Medien verbreitet werden. Ohne näher darauf einzugehen, wurden hier nur einige wenige vorgestellt und auf die veränderten Bedingungen im digitalen Zeitalter hingewiesen.

Sowohl den VertreterInnen von Verschwörungstheorien wie auch ihren KritikerInnen geht es um die Wahrheit. Beide Gruppen werfen der jeweils anderen vor, Fake-News zu verbreiten. Und natürlich gibt es kaum einen Verschwörungstheoretiker, der diese Bezeichnung für sich selbst akzeptieren würde. Wie in diesem Artikel beschrieben, handelt es sich also um eine Fremdzuschreibung. Oft bleibt es eine Frage der Definitionsmacht, was bereits als Verschwörungstheorie gilt und was noch als realistische Nachricht oder sogar als seriöse Wissenschaft vertreten wird (cf. Muth 2018). Verschwörungstheoretiker sind die „Anderen“, die nicht mit der eigenen Weltsicht übereinstimmen. Die eigene Wahrheit wird oft als die einzig mögliche wahrgenommen, auch wenn sie sich für andere absurd oder sogar verrückt anhören mag.

Dieses Merkmal teilen Verschwörungstheorien mit den meisten Religionen und hat primär mit dem Glauben an etwas zu tun, was weder zu beweisen noch einfach zu widerlegen ist. Die Faszination, die von Verschwörungstheorien ausgeht und über die auch in diesem Artikel berichtet wurde, hängt vor allem mit der Möglichkeit zusammen, dass sie sich als real erweisen könnten oder mögliche Geheimnisse offenbaren (cf. Muth 2018).

Wie eine hier zitierte Studie betont, geht es um das Verstehen und Begreifen von schwer erklärbaren Phänomenen bzw. Ereignissen. Oft werden auf schwierige Fragen und komplexe Zusammenhänge die einfachsten Antworten akzeptiert. Trotzdem steht dahinter der Versuch, den eigenen Horizont zu erweitern. Deshalb bleiben Verschwörungstheorien Wahrheitskonstruktionen nach einer erkenntnistheoretischen Intention.

Es gibt keine Garantie, weder über die Medien noch über wissenschaftliche Quellen, die vollständige Wahrheit vermittelt zu bekommen. Das liegt in der Regel nicht an einer bösen Absicht oder einer „Verschwörung“, sondern an der subjektiven Wahrnehmung, wie auch der Konstruktivismus betont. Trotzdem dürfen historische Fakten nicht ignoriert werden, die von der Postmoderne manchmal mit einer bloßen Meinung verwechselt wurden. Beispielsweise gilt es als 
bewiesenes Faktum, dass der Irak über keine Massenvernichtungswaffen verfügte, als er aufgrund dieser von beinahe sämtlichen westlichen Leitmedien vertretenen Behauptung 2003 von den USA und ihren Verbündeten bombardiert wurde.

Die Hinterfragung des Mainstreams darf daher nicht als Tabuverletzung betrachtet oder automatisch als Verschwörungstheorie bezeichnet werden. Denn das Schärfen der eigenen Kritikund Urteilsfähigkeit wird zur alternativlosen Voraussetzung, um künftigen Verschwörungstheorien und Fake News begegnen zu können.

Nach dem Ausbruch der so genannten Corona-Krise erhält das Thema eine neue Aktualität. In Zeiten der Verunsicherung und der Krise gibt es nicht nur eine aktive Zunahme des Medienkonsums und der Nachrichtenproduktion, sondern auch der Verschwörungstheorien und der "alternativen Fakten". Über die Fragen, woher das Virus stammt, wo es zuerst warum aufgetreten sei und wie sich die Pandemie weltweit ausbreiten konnte, bestehen die unterschiedlichsten Theorien und Thesen. Besonders in den sozialen Medien und im Internet kursieren Artikel, Videos und damit unzählige Gerüchte zum Thema. Im Netz konnten sich die verschiedenen ExpertInnen austauschen oder sich vielmehr gegenseitig widersprechen.

Für die Rezipientinnen ist es aktuell noch schwieriger geworden, auf die Nachrichtenflut zu reagieren und sich zu orientieren: Welche Informationen und Ratschläge sind hilfreich, welche sind einfach fahrlässig oder sogar gefährlich, welche dienen eher als Material für Satire und Unterhaltung? Die Krise hat gezeigt, wie schnell die Menschen sich an neue Verhältnisse anpassen können und auch bereit sind, ihre politischen Freiheiten aufzugeben und sich neuen Maßnahmen zu unterwerfen, wenn sie sich davon etwas mehr Sicherheit versprechen. Sie verfolgten die Berichterstattung über die unaufhaltsame Ausbreitung und ordneten sich den neuen Regelungen unter, deren Sinnhaftigkeit entweder erkannt oder erhofft, auf jeden Fall aber nicht in Frage gestellt wurde. Unabhängig davon, um welches Land oder welches politische System es sich handelte, waren global ähnliche Reaktionen zu beobachten. Ausgangsbeschränkungen und Kontaktverbote, Maskenpflicht und der Lockdown mit katastrophalen Folgen für die Wirtschaft. Neue Methoden der Überwachung und Kontrolle wurden eingeführt, etwa digitale Apps, die Bewegungsprofile und Kontakte registrieren sollten. Erst leise und vorsichtig, aber mit der Dauer der Maßnahmen immer lauter mehrten sich vor allem in den sozialen Medien die Stimmen, die vor einem Missbrauch der Situation warnten und der offiziellen Darstellung widersprachen.

Die jeweiligen Regierungen reagierten unterschiedlich darauf, verurteilten aber einheitlich jede alternative Darstellung der offiziellen Version und den Anleitungen, wie sie von der WHO und den anerkannten Institutionen verbreitet wurden. Dass auch diese Stellen und der Einfluss von privaten Stiftungen und der Pharmaindustrie kritisiert wurden, betrachteten viele als zusätzliche Bedrohung. In Österreich und anderen europäischen Ländern wurden eigene Stellen (eine eigene vom Innenministerium eingerichtete Task force) eingerichtet, deren Aufgabe es ist, das Internet und die Medien nach Fake-news zu untersuchen. (Bundeskanzleramt 2020) Auch die bekanntesten sozialen Medien haben sich dazu bereit erklärt, ihre Seiten $\mathrm{zu}$ kontrollieren und von Falschmeldungen zu säubern. Begründet wurde das mit der Gefahr, dass abweichende Erklärungsmuster dazu führen könnten, dass wichtige Regeln nicht eingehalten würden und sich das Virus damit schneller ausbreiten könnte. Vereinzelt kam es auch $\mathrm{zu}$ bedrohlichen Therapievorschlägen, die Leib und Leben von Menschen gefährdeten.

Allerdings widersprachen sich auch die EpertInnen untereinander als auch sich selbst, mit jeder neuen Erkenntnis, die zum Umgang mit dem Virus gesammelt wurde. Die damit einhergehende Glaubwürdigkeitskrise der offiziellen Stellen bewirkte eine Zunahme von abweichenden Meldungen. Der in dieser Arbeit beschriebene Kreislauf von offiziellen und abweichenden Nachrichten wurde also eher noch stärker angeregt als dass er eingedämmt worden wäre. Diese neuen Entwicklungen 
zeigen, dass es eine neue Diskussionskultur braucht, die nicht einfach auf Verordnungen und Repressionen aufbaut.

Die neuen Maßnahmen erinnern an George Orwells "Wahrheitsministerium" und haben das Vertrauen in die offiziellen Stellen nicht gerade erhöht. Der Vorwurf von Zensur steht im Raum. Zu Meinungspluralismus und Transparenz gibt es keine Alternative; auch würde es den etablierten Mainstream- und Qualitätsmedien nicht schaden, selbstkritisch die eigenen Irrtümer und die unkritisch von den Nachrichtenagenturen übernommenen Artikel zu reflektieren. Das würde der eigenen Reputation nicht schaden, sondern könnte dazu beitragen, wieder an Vertrauen zu gewinnen. Nur so könnte verhindert werden, dass die Menschen unseriösen Medien und Verschwörungstheorien mehr Glauben schenken als den um Objektivität bemühten Journalistinnen und Journalisten.

\section{Literatur}

APA/ Reuters (13.01.2015): "Lügenpresse" ist Deutschlands Unwort des Jahres:

https://www.diepresse.com/4637372/lugenpresse-ist-deutschlands-unwort-des-jahres [04.11.2019]

Balibar Etienne (2006): Der antisemitische Komplex-Eine selbstkritische Konzeption des Feindes: Zur Doppelgestalt von Judenhass und Araberhass, (First Edition: Frankfurter Rundschau, 25.06.2002): Der.Wish05-Zeitschrift für Vielseitige, Wien

https://www.bundeskanzleramt.gv.at/bundeskanzleramt/nachrichten-derbundesregierung/2020/raab-kampf-gegen-fake-news-in-16-sprachen.html [20.04.2020]

BR-Mediathek (11. 09. 2019): Verschwörungstheorien - wer glaubt denn sowas?

https://www.br.de/mediathek/video/stationen-11092019-verschwoerungstheorien-wer-glaubt-dennsowas-av:5d416cab7c69d4001a545b54 [08.11.2019]

EU Projekt: „Analyse von Verschwörungstheorien“

Buchmayr, Florian/ Köhler, Jonas (2016/2017): Tutorium-Abschlussbericht: Die gesellschaftliche Konstruktion der Verschwörung, Kultur- Sozial- und Bildungswissenschaftliche Fakultät/ Kulturwissenschaftliches Institut, Humboldt-Universität Berlin

Central Intelligence Agency (CIA) (22.04.2019 a): Freedom of Information Act - Electronic Reading Room:

https://www.cia.gov/library/readingroom/ [15.11.2019]

CIA 1990: UNIDENTIFIED FLYING OBJECTS (UFO) IN ANCIENT CHINA

https://www.cia.gov/library/readingroom/document/0000042351 [12.11.2019]

CIA 1967: UFO BRIEFING FOR DR. EDWARD CONDON, 5 MAY 1967:

https://www.cia.gov/library/readingroom/document/0000015436 [04.11.2019]

CIA 2017: Reading room: UFO:

https://www.cia.gov/library/readingroom/search/site/UFO [04.11.2019]

CIA 2019 b: Reading room: FOIA

https://www.cia.gov/library/readingroom/search/site/MK\%20Ultra [04.11.2019]

Gesundheit.de (16.03.2017) Wie viel Eisen ist wirklich im Spinat?: 
https://www.gesundheit.de/wissen/haetten-sie-es-gewusst/ernaehrung/wie-viel-eisen-ist-wirklichim-spinat [15.11.2019]

Hofbauer, Hannes (2001): Balkankrieg - Zehn Jahre Zerstörung Jugoslawiens, Promedia Verlag, Wien

Huber, Matthias (19. November 2016): Facebook: Zuckerberg verspricht Maßnahmen gegen Fake News:

https://www.sueddeutsche.de/digital/facebook-zuckerberg-verspricht-massnahmen-gegen-fakenews-1.3257346 [02.11.2019]

Johnson N.F. et al (21.08.2019): Hidden resilience and adaptive dynamics of the global online hate ecology, Nature 573, p.261-265:

https://www.nature.com/articles/s41586-019-1494-7 [06.11.2019]

Krammer, Hubert (2015): Die Medien und der Krieg, in: Arbeitskreis Süd-Nord (2015):Lüge macht Krieg, Theorie und Praxis Verlag, Hamburg 27-40

Khella, Karam (1995): Universalistische Geschichtstheorie, Theorie und Praxis Verlag, Hamburg

Landeszentrale für politische Bildung (2019): Verschwörungstheorien - Warum sind sie so erfolgreich und was kann man tun?

https://www.lpb-bw.de/verschwoerungstheorien.html [04.11.2019]

Miguel de la Riva (2. November 2019): Soziologe: "Verschwörungstheorien reflektieren oft Unterdrückungsverhältnisse", Interview im online-Standard mit Didier Fassin:

https://www.derstandard.at/story/2000110437146/soziologe-verschwoerungstheorien-siereflektieren-haeufig-unterdrueckungsverhaeltnisse-und-aengste [03.11.2019]

Muth, Max (6.6.2018): Warum Verschwörungstheorien nicht tot zu kriegen sind: https://www.tagesanzeiger.ch/kultur/diverses/WahreVerschwoerungstheorien/story/26337873 [04.11.2019]

Özkececi, Zehra (2018): Demokratie auf dem Prüfstand - Demokratie und Medien in der Türkei, Südwestdeutscher Verlag für Hochschulschriften, Beau Bassin

Schmid, Fabian/ Muzayen, Al-Youssef (28. Februar 2019): Verschwörungstheoretiker dürfen Wikipedia-Autor outen:

https://www.derstandard.at/story/2000098702546/verschwoerungstheoretiker-duerfen-wikipediaautor-outen [16.11.2019]

Schwarz, Carolina (8. 12. 2016): Verschwörungstheorie über Clinton- \#Pizzagate geht weiter:

https://taz.de/Verschwoerungstheorie-ueber-Clinton/!5364337/ [03.11.2019]

Standard-Redaktion, (02.11.2019): Trollfabrik aufgeflogen: Politische Werbung mit Fake-Postings:

https://www.derstandard.at/story/2000110601445/trollfabrik-aufgeflogen-politische-werbung-mitfake-postings [04.11.2019]

The 9/11 Commission Report (2004):

https://govinfo.library.unt.edu/911/report/911Report.pdf [18.11.2019]

Wieselberg, Lukas (21.08.2019): Mit Mathematik gegen Onlinehass, science.ORF.at: https://science.orf.at/stories/2990262/ [15.11.2019] 
Zeit-online Redaktion et al (01. 05. 2019): Große Medienhäuser einigen sich auf Kodex für Christchurch-Prozess:

https://www.zeit.de/gesellschaft/zeitgeschehen/2019-05/neuseeland-christchurch-anschlag-prozesskodex-berichterstattung [15.11.2019] 